\title{
Using finite element analysis to influence the infill design of fused deposition modelled parts
}

\author{
James A. Gopsill ${ }^{1}$. Jonathan Shindler² Ben J. Hicks ${ }^{2}$
}

Received: 5 May 2017 / Accepted: 21 November 2017 / Published online: 28 November 2017

(c) The Author(s) 2017. This article is an open access publication

\begin{abstract}
Additive manufacturing (AM) has and continues to experience considerable market and technological growth with many forecasting a tripling in market value over the next decade. One of the primary drivers for this growth is the increased freedom afforded to the design of both the external form and internal structure of fabricated parts. This freedom presents greater opportunities in optimising a parts mechanical properties, (such as strength, stiffness and mass), which in turn leads to enhanced performance whilst potentially reducing material use and hence, environmental impact. Realising this potential will further increase the viability of AM for a greater range of engineering contexts. Correspondingly, the contribution of this paper lies in the creation and validation of a method for the topological optimisation of the infill structure of fused deposition modelled (FDM) components. The proposed method uses results attained from finite element analysis (FEA) to influence the design of the internal structure (i.e. infill) by locally varying the composition of the infill based upon the associated stress values. This paper presents and discusses the proposed method, and demonstrates the generalisability of the method through its ability to handle complex geometries and loading conditions, and manufacturing process constraints. In addition, the paper validates the method through testing of FDM beams comprised of FEA influenced and standard honeycomb infill designs undergoing four different loading scenarios. The validation reveals that a three and a half times increase in strength can be achieved where the stress profiles are well defined within the structure. In addition, the FEA-influenced beams exhibited more consistent failure mode profiles, which maybe desirable for designing parts with specific failure mode characteristics.
\end{abstract}

Keywords Topology optimisation · Internal geometry $\cdot$ Infill $\cdot$ Internal structure $\cdot$ Fused deposition modelling (FDM) Finite element analysis (FEA)

\section{Introduction}

Additive manufacturing (AM) continues to experience considerable market and technological growth with forecasts indicating a tripling in market value over the next decade [1]. In this market, fused deposition modelling (FDM) is one of the most commonly applied techniques [2] and has been used in a variety of contexts including:

- supporting and facilitating engineering degree courses;

- creating novel structures for bio-medical research;

James A. Gopsill

j.a.gopsill@bath.ac.uk

1 Department of Mechanical Engineering, University of Bath, Bath, UK

2 Department of Mechanical Engineering, University of Bristol, Bristol, UK
- highly customised and individualised consumer products; and,

- rapid prototyping products in engineering design consultancies.

One of the main affordances of AM is the capability to produce highly complex external and internal (infill) geometries that enable engineers to optimise the deposition of material for a given application. This provides benefits in terms of reducing component cost through a decrease in both material use and manufacturing time. It is also a common goal for manufacturers to optimise the performance of their parts through their strength-to-weight ratio. The prevalence of which is exemplified by the extensive literature on the subject of topology optimisation (see, for example, [3-5]).

Although many techniques and algorithms exist for defining the theoretical optimum topology of a component, the ability to translate the optimised geometry into 


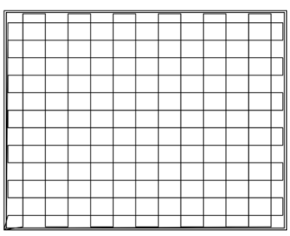

(a) Linear

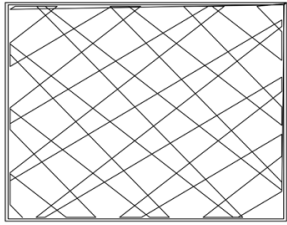

(f) Line

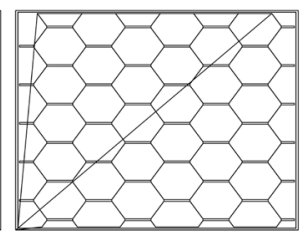

(b) Hexagonal

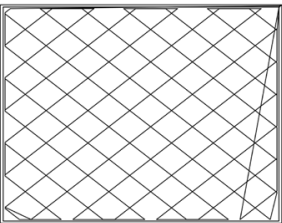

(g) Rectilinear

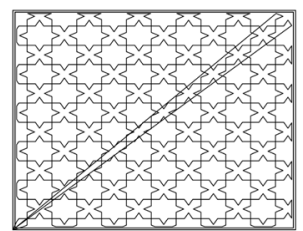

(c) Moroccan Star

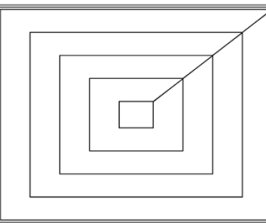

(h) Concentric

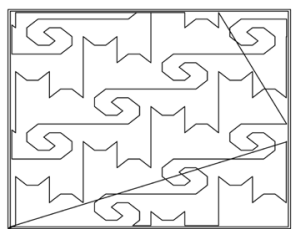

(d) Catsfill

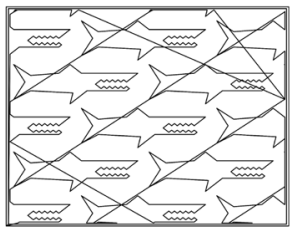

(e) Sharkfill

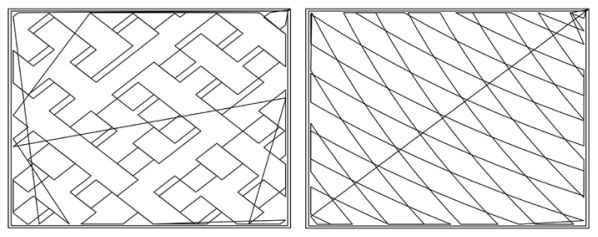

(i) Hilbert Curve (j) Archimedean Chords

Fig. 1 Infill strategies from MakerWare (a-e) and Slic3r (f-j) software

a manufacturable component continues to be a challenge. This is due to the constraints imposed by the manufacturing techniques being applied. In the case of selective laser sintering (SLS), research has already begun to overcome these challenges. In comparison, the development of this underlying capability for FDM remains relatively immature [6-8]. This is in part due to the differing manufacturing constraints between the processes preventing the same topology optimisation techniques being applied.

In contrast to SLS, where the process involves the layering of granular material and sintering at specific locations, FDM involves the layer-by-layer deposition of material and this introduces the challenge of defining the deposition path as the layers are highly dependent upon the previous to provide support for the material being extruded. Rafts, supports and bridging are all techniques that have been introduced to enable the manufacture of geometrically complex parts using FDM. It is argued that the very nature of extruding material is a key-contributing factor to why two-dimensional repeating patterns are commonly applied for the infill of FDM components (Fig. 1). This is alongside the reduction in computational time to produce the part as the pattern is consistent across layers and thus, only the intersections between the pattern and model surfaces have to be calculated. In addition, the infill percentage can be easily controlled by scaling the pattern.

Given the extensive literature on topology optimisation and the ability to manipulate the internal structure of FDM parts, it is contended that there is potential to influence the infill design of a part using finite element analysis (FEA) results whilst also considering the manufacturing constraints of FDM and not effecting the external form of the component. Factoring the manufacturing constraints within design tools has been highlighted as a key requirement in empowering the development of innovative products [9]. And, even though this will not lead to a truly optimised structure, it is argued that significant improvements can be attained in terms of the strength-to-weight ratio of the component. In addition, the components' external form may be crucial to its function, such as brand representation [10]. By providing an optimisation process that can meet these constraints will lead to further democratisation of the design process by enabling human-machine co-creation, where the engineer focuses on the external geometry and the machine focuses on the internal geometry.

Consequently, this paper describes a process that uses the results from FEA to influence the automatic generation of a components infill. The paper first discusses related work concerning the design and manufacture of AM parts, which has primarily focused on the accuracy and mechanical properties of parts, and design of infill patterns. The paper then continues by discussing the proposed process of using FEA to influence the design of the infill where the focus has been on manufacturability, generalisability and automation. To evaluate the proposed method, a comparison of the stress-strain profiles of parts with hexagonal and FEA-influenced infill has been conducted. The paper then concludes by expressing the key findings of this method as well as discussing potential areas of future work.

\section{Related work}

To date, the majority of research concerning the optimisation of fused deposition modelled (FDM) parts has focused on either the accuracy of the manufactured part compared to the digitally designed model, or strength of the part given changes to the parameters within common slicing approaches (for example, infill percentage, infill orientation and layer thickness). 


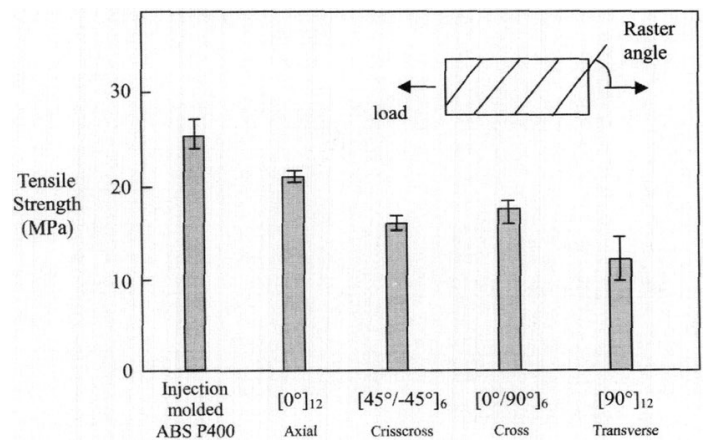

Fig. 2 Tensile strength of FDM ABS compared to injection moulded (from: [11])

The accuracy of the part to its digital counterpart has been analysed by Sood et al. [12] who applied Taguchi's design of experiments on five common parameters ${ }^{1}$ of the FDM process and determined that the shrinkage phenomena is most influential. This has been confirmed by Lanzotti et al. [13] in their study of the RepRap open-source 3D printer, which revealed that layer thickness and the consequent shrinkage was the leading factor in attaining geometrically accurate parts. It was also revealed that to optimise accuracy in all Cartesian co-ordinates generally necessitates a compromise as optimising in a single direction negatively impacts on the others. Further, analysis of part accuracy by Pennington et al. [14] highlighted that part position on the bed space, part size and temperature of the work area also contribute significantly to accuracy. To improve the accuracy of the printed part, Gregorian et al. [15] found that the optimum shrinkage compensation factor to be applied to the manufacturing process was 1.007 for their particular machine. Whilst work by Zhang and Chou [16] have looked to model the shrinkage phenomena through FEA to automatically generate the compensation factors for FDM machines. In addition, Pandey et al. [17] show that improvements to accuracy and quality can be made through adaptive layer height control in the generation of the tool-head path. This highlights the potential to optimise part geometry through adjustment of the numerical control rather than the machine itself.

In terms of investigating the mechanical properties of parts produced by FDM, it has been shown that the deposition process itself has profound anisotropic effects on the part, which leads it to behave much more like a laminate [20, 21]. Ahn et al. [11] show that a decrease of $80 \%$ in tensile strength can occur if the material is deposited in an inappropriate orientation (Fig. 2). This has since led to the development of algorithms to automatically determine the optimum orientation for a given part [22]. In addition, a number

\footnotetext{
${ }^{1}$ layer thickness, air gap, raster angle, orientation and raster width
}

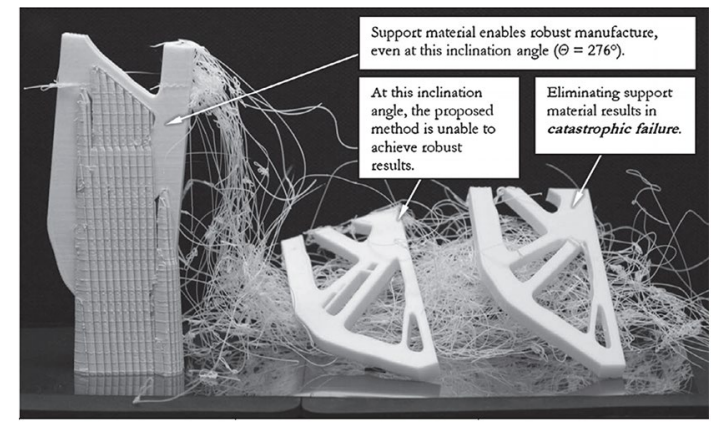

(a) Applying SLS optimised topology optimisation techniques on FDM machines (From: [18])

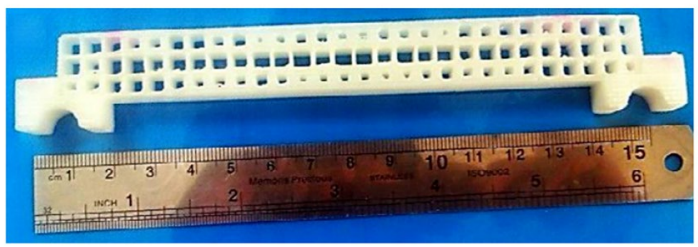

(b) Localised filling of linear mesh of FDM parts (From: [19])

Fig. 3 Topology-optimised parts using AM technologies

of studies have examined tensile and flexural strength of FDM parts with all results corroborating the fact that layer thickness is the most influential factor [23-27]. However, decreasing the layer height to increase strength has a negative impact on the surface roughness. Thus, a compromise must again be sought. In addition, reductions in layer height can increase factors such as distortion, layer cracking and de-lamination leading to premature part failure [23]. While Jones et al. [24] highlight that Acrylonitrile Butadiene Styrene (ABS) has been shown to lose $20 \%$ of its strength when extruded using FDM whilst polylactic acid's (PLA's) strength remains unaffected.

Initial research into topology-optimised FDM structures attempted to emulate SLS techniques with Leary et al. [18] discovering that considerable use of rafts and supports were required to produce the component (Fig. 3a). And even with these additions, there was a high likelihood of the part failing during manufacture. In addition, those that were manufactured successfully produced a considerable amount of waste material and required significant post-processing time in terms of removing the support material. In contrast, Rezaie et al. [19] and Lei et al. [28] have developed techniques that locally varies the thickness of the linear mesh within a FDM part (Fig. 3b). Their results show that the approach can lead to potential gains of up to $25 \%$ in the strength-to-weight ratio of the component.

This growing interest in the optimisation of internal geometry based on the predicted loading scenario has also been investigated by Gopsill and Hicks [6], who demonstrate that a 79\% increase in the stiffness of a part can be attained through the alignment of infill to the direction of stress 

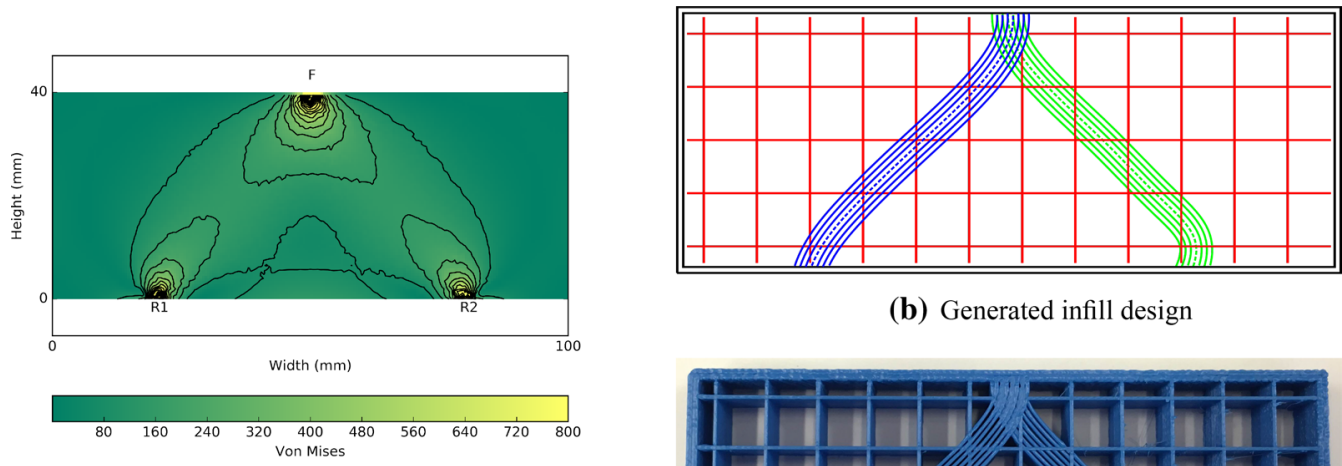

(b) Generated infill design

(a) Predicted stress profile

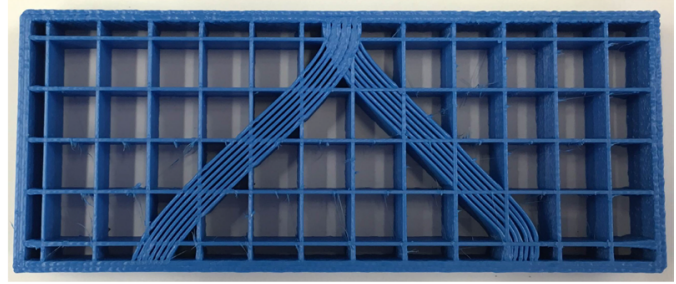

(c) Manufactured beam

Fig. 4 Infill designs generated from the predicted planar stress profiles of parts (from: [6])

(Fig. 4). In addition, the topology-optimised parts showed more consistent buckling and failure mode behaviour. This phenomena gives rise to the potential for designing parts that provide visual indicators of structures exceeding their designed loading and/or sacrificial parts that prevent components from being damaged if an engineering system operates outside its normal range. Although the potential benefits have been demonstrated, the process is still limited to planar stress profiles and has yet to be evaluated for more complex loading scenarios.

In summary, the related work reveals that the majority of current research has focused on optimising the manufacturing process with regards to enhancing the accuracy, tolerance, print time and material use. In addition, there is a growing trend towards studies that provide processes that optimise the topology of a component. Supporting the design of components through FDM remains an emerging field with many potential areas that could be explored. In particular, it is contended that the results from modelling techniques, such as FEA, have the potential to positively influence the internal geometry of a component. Consequently, the contribution of this paper is the presentation of a method that uses the results from FEA to influence the generation of the internal geometry of FDM parts.

\section{Influencing the infill design using FEA}

The process for influencing the infill design for generic FDM parts is presented in Fig. 5 alongside an illustration of the outputs from the various stages in Fig. 6. The key objective of the process is to use the results from FEA to influence the infill design whilst ensuring the strategy takes the manufacturing constraints of FDM into account, does not alter the external geometry, is applicable to as many components as possible, and requires minimal user intervention. The strategy comprises of five stages:

1. Model construction

2. Determining mesh size and perimeter identification

3. Linear infill design

4. FEA-influenced infill design

5. G-code generation

The first stage consists of the construction of the stereolithography (STL) file detailing the geometry of the part alongside the exported results from a Finite Element model in the form of a comma-separated variable (csv) file (Fig. 6a, b). It is important to note that the role of the FEA is to guide the design of the infill in stage four and does not reflect the actual stress within the final structure. Once constructed, the process can continue to stage two where the optimum internal mesh sizing is derived. This is followed by a loop that generates the relevant G-Code for each deposition layer. The start of each deposition layer involves the identification of the geometry perimeter (Fig. 6c) and the generation of the related number of shells that will be produced. Shells being the numbers of times the FDM machine will deposit material to form the perimeter of the part where an offset is applied to ensure no overlapping of material occurs (Fig. 6e).

Once completed, the process moves to the third stage which generates the linear infill design. The objective of the linear infill is to provide a consistent structure that the FEAinfluenced infill design (stage four) can attach and bridge across (Fig. 6g). This is an important feature as it enables the FEA-influenced infill designs to alter as the layer height 


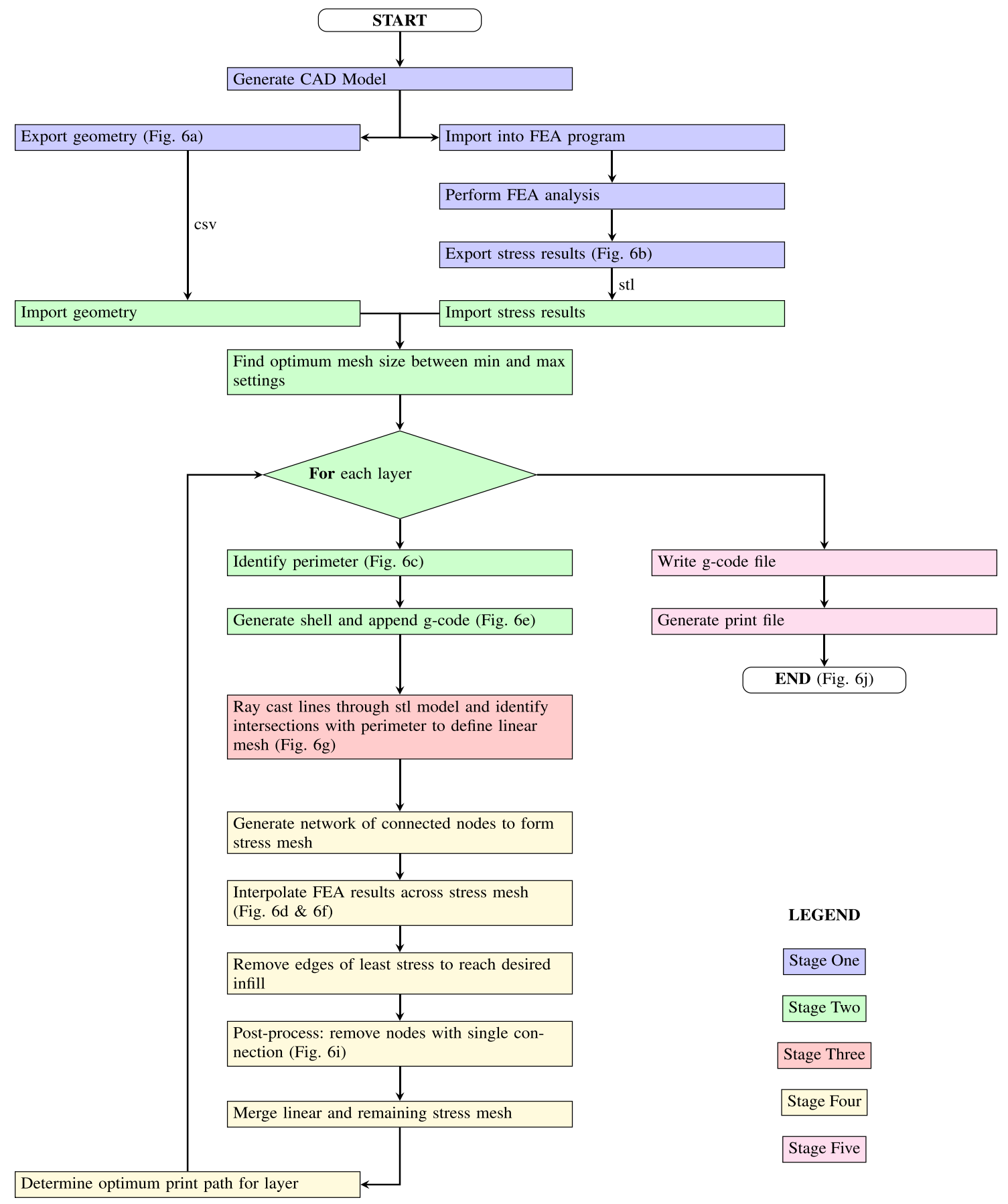

Fig. 5 Infill designs workflow

increases and thus, provide appropriate support for threedimensional loading scenarios (this is demonstrated and later discussed in Fig. 13). The linear infill is also used to counter the potential of introducing instabilities and unsupported features by not placing material within low stress areas [3].

Stage four continues the development of the deposition layer by generating the FEA-influenced infill design. This is achieved by extending the linear infill design to form a lattice design. Although limiting the topology to a lattice design will prevent the strategy from producing truly optimal solutions, lattice structures have been selected due to their:

- proven capability to transfer and withstand high loading; 


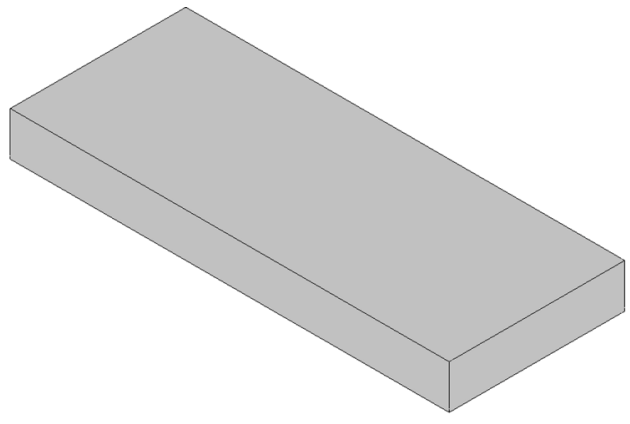

(a) STL model

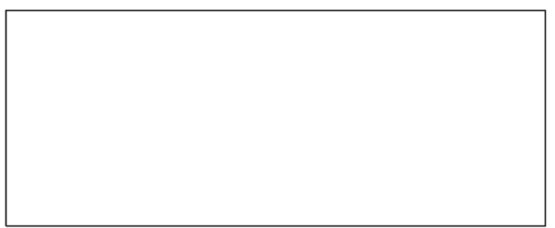

(c) Perimeter identification at deposition layer

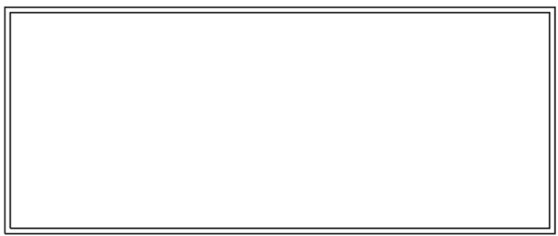

(e) Shell generation

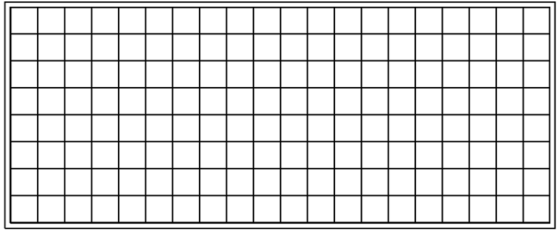

(g) Linear infill design

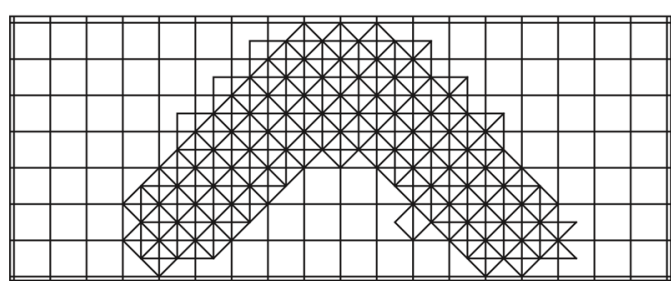

(i) Combined infill design

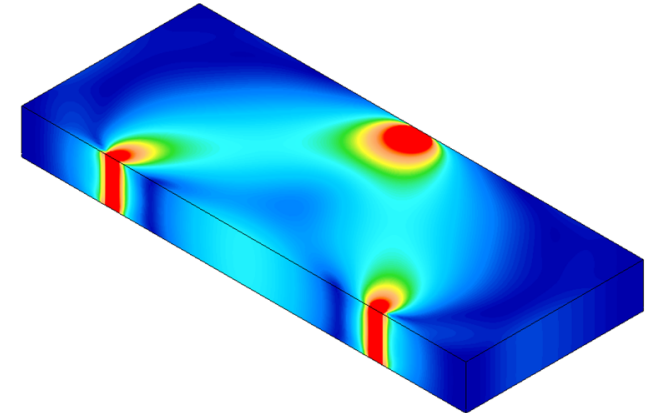

(b) FEA model

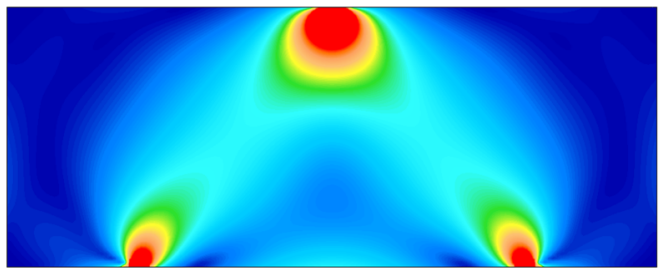

(d) FEA results at deposition layer

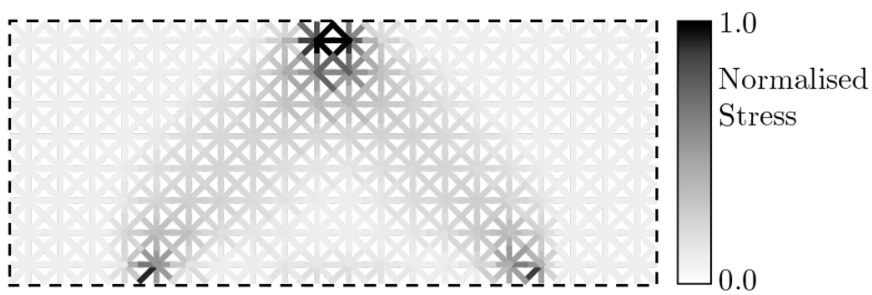

(f) FEA influences lattice elements

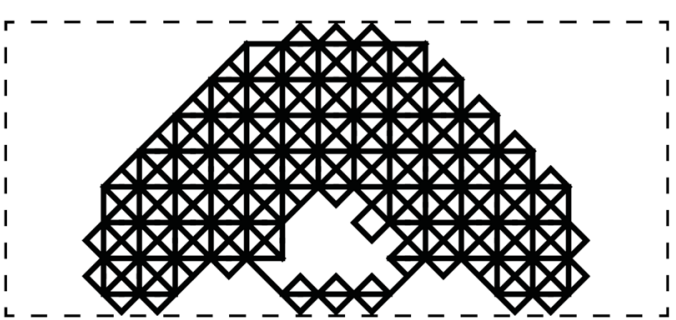

(h) FEA influenced infill design

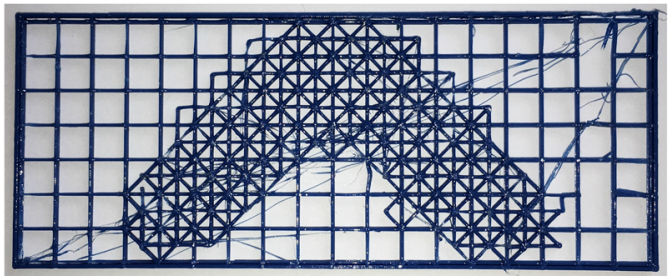

(j) Printed design

Fig. 6 Illustrative examples of the stages taken to generate an infill design that has been influenced by FEA

- highly connected nature that enables bridging, which is important as the optimised internal structure may evolve layer-on-layer (c.f. Fig. 13);

- ability to be reliably manufactured using FDM;
- ability to be fitted to complex external geometries of FDM components; and,

- generation being computationally inexpensive when compared to more complex geometries such as the opti- 
Fig. 7 Examples geometries for strategy

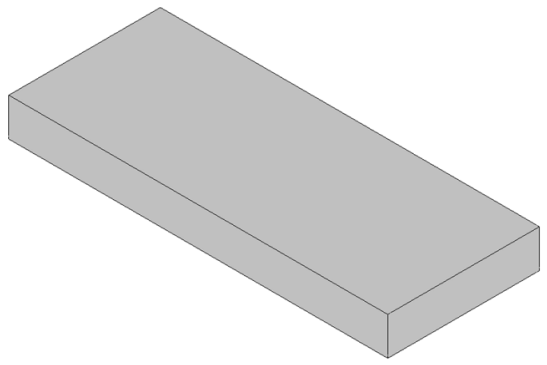

(a) Beam

Fig. 8 Finite element analysis of beam and bracket

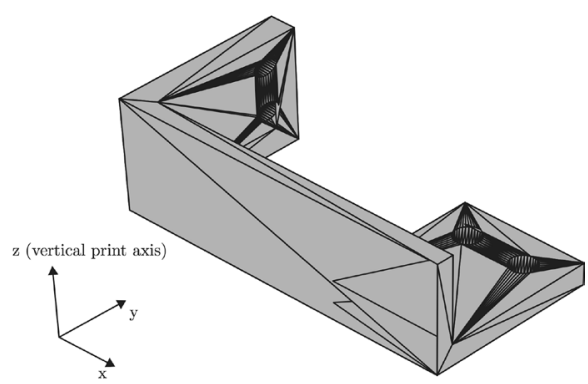

(b) Bracket

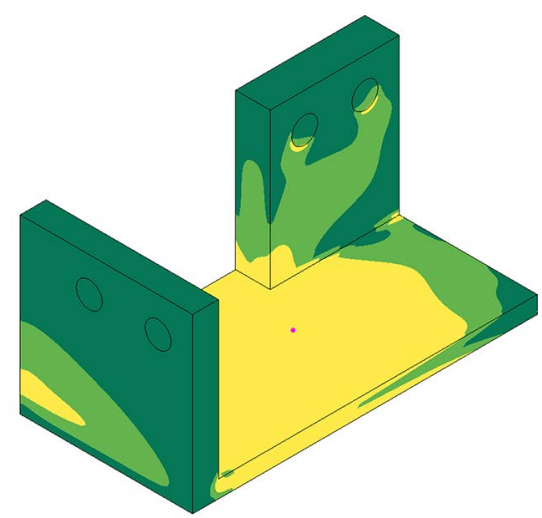

(b) Bracket mal positioning of bezier splines along force pathways [6].

The local stress values are then mapped to the lattice elements (Fig. 6f) and a removal of elements with least stress occurs until the desired infill percentage is met (Fig. 6h). Following this, a post-processing of the infill occurs where the objective is to identify and remove elements that are unable to support any loading as they terminate without connecting to the rest of the structure.

Upon completion of the post-processing, merging of the FEA influenced and linear infill occurs and involves the union of the two designs. The final aspect of Stage Four is to then define the optimum print path for the resulting infill design to reduce the time taken to manufacture the part. The process then progresses to Stage Five, where the G-Code is written and is either used directly in the printing process or further processed into the relevant binary print file.

The following sections provide further details of each stage alongside an example of a beam being optimised for a three-point bend test, and a bracket in a contrived 3-dimensional stress scenario. The STL geometries for both examples are shown in Fig. 7. The beam has been chosen as it is used later to evaluate the process in terms of the potential performance improvement. Whilst the bracket is considered to demonstrate the generalisability of the process. In particular, the bracket geometry is non-uniform along the axis of print, contains multiple-perimeter geometry (for example, holes) and has been placed under a three-dimensional loading scenario.

\subsection{Model construction (stage one)}

The initial step is to create the computer-aided design (CAD) model of the part to be optimised. From this model, both the STL file for the identification of the part perimeter and the FEA model are generated. In the case of this paper, AutoDesk Inventor 2015 and AutoDesk Simulation Mechanical 2015 were used.

The process uses the FEA model to perform an evolutionary structure optimisation (ESO) of a continuous structure within a design domain $[29,30]$. The design domain being the model geometry with the loads being applied and the premise is to identify the load paths through the continuum structure. These load paths highlight the areas where it is desirable to maintain a high concentration of material and the non-load paths are the areas where material could be reduced or removed.

Figure 8a shows the von Mises stress calculated by the FEA analysis for a three-point bend test with the contours highlighting lines of constant stress. It can be seen that there are two main load paths that both emanate from the applied 
Fig. 9 Mesh size and perimeter identification

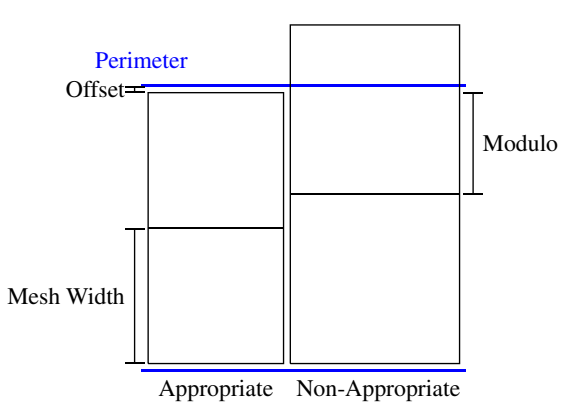

(a) Determining the appropriate mesh sizing

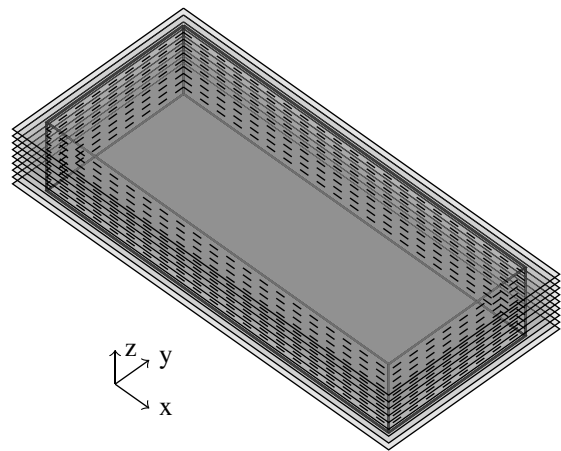

(b) Cutting planes through the beam STL load and go directly to the points of reactions at the pinjoints. Less obvious, although visible due to the contours, is an area of high stress on the underside of the beam, between the two reaction locations. As the beam is under high compressive loads, this area is undergoing significant bending stress that also needs to be taken into account in the optimisation of the beam structure. It is also interesting to note that the stress contours are almost but not completely symmetrical even though this is the expectation. This can be attributed to the irregularities in the mesh construction of the FEA and accuracy of the boundary conditions. In many cases, FEA analysis is used as a guide to show potential areas of highstress within a component and real-world validation is often required to ensure that it accurately reflects the real-world product. In this case, the FEA is used as a guide to influence the infill design and thus, does not have to be a fully validated model. Figure $8 \mathrm{~b}$ demonstrates the three-dimensional loading case of the bracket that is later used to explore the generalisability of the proposed process.

The results from the FEA analysis, which include the Cartesian co-ordinates of the nodes and their associated stress values are exported as a csv file. Both the STL and csv file are then imported into the script to generate the influenced infill design for the part.

\subsection{Mesh size and perimeter identification (stage two)}

To generate the internal structure of the part, it is first necessary to establish an appropriate mesh sizing before continuing into the loop where the infill design for each deposition layer is generated. This is a common task that all slicing routines perform ahead of generating the infill design for the component.

To establish the mesh sizing for the print file, the process attempts to ensure that the perimeter of the part interfaces with a complete mesh square of the infill (as demonstrated in Fig. 9a). To achieve this, the user manually selects a minimum and maximum threshold for the mesh sizing, and the process discretises this range and calculates the modulo in both the $x$-and $y$-directions for the range of mesh sizing. The $x$ and $y$ lengths are determined by the minimum and maximum values for each direction within the STL model alongside an offset to ensure the inner mesh does not interfere with the perimeter when being printed. Once the modulo for both dimensions are calculated, these are summed and form the error for the given mesh sizing. The mesh sizing that minimises the error. This optimisation works particularly well for flat faces along the Cartesian co-ordinates but has not been fully evaluated for curved surfaces.

Once the mesh sizing has been determined, the process can now iterate through each deposition layer and generate the deposition path. For each layer, there is a need to identify the perimeter of the part. This has been achieved through the use of the visualisation tool-kit (VTK) Python library [31]. A slicing plane is generated using the library and the intersections between the plane and the STL model are identified (Fig. 9b). A set of lines is generated from where the plane intersects the individual polygons of the STL file and it is then the case of identifying the connecting path between the lines. This is achieved by taking a single line and identifying the neighbouring line by finding the end points that match. The process then continues to the end of the next line and repeats until the sequences of lines returns to the starting line. This forms a perimeter polygon for the part. There is then a check to see whether any lines remain that do not form part of the chain. If so, a new chain is generated with the remaining lines. This cycle repeats until all the lines are associated to a chain. This enables the identification of complex geometry with multiple perimeters such as a part containing holes and/or cut-outs, such as that depicted in Fig. 10.

\subsection{Linear infill design (stage three)}

The third stage of the process involves the generation of a set of uniformly distributed vertical and horizontal lines to form the linear infill design for the part. The linear infill exists to provide a consistent structure for the FEA-influenced infill design to attach to. As the 
Fig. 10 Identifying multiple perimeter geometry during slicing

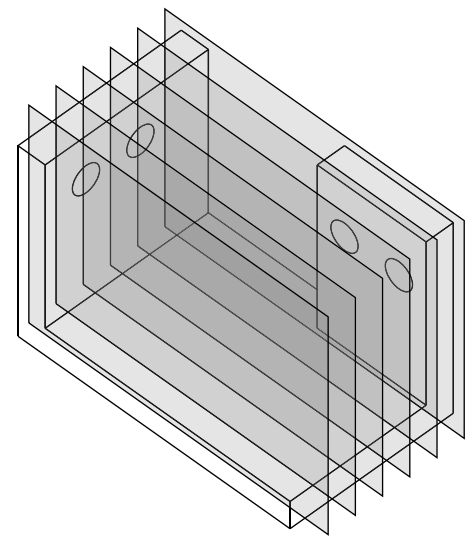

(a) Slicing of bracket geometry

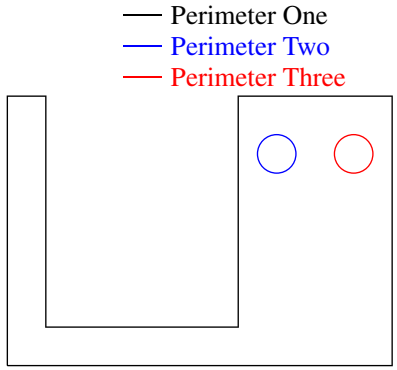

(b) Multi-perimeter identification
Fig. 11 Generating a linear mesh across a deposition layer

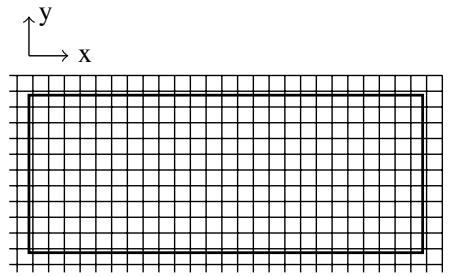

(a) Beam Ray Casting

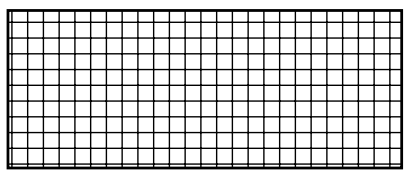

(c) Beam Linear Mesh

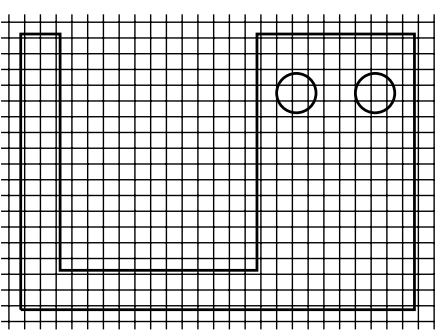

(b) Bracket Ray Casting

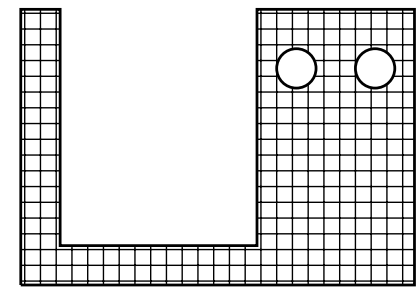

(d) Bracket Mesh
FEA-influenced infill design may change with the layer height, the linear infill enables bridging to occur and thus, ensures the material is deposited in the desired locations.

The linear infill generation utilises the mesh offset and mesh width variables defined in Stage Two, and the ray casting functionality of VTK. The strategy generates rays along the $x$ - and $y$-axes for the given layer height, and identifies the intersections of rays against the STL model of the part (Fig. 11a, b). For complex geometries, the rays have the potential to intersect the model multiple times and thus, the lines of interest are determined by pairing intersecting points as the ray passes through the model (i.e. points 1 and 2 will form a path and the same goes for 3-4, 5-6, etc...). Fig. 11 illustrates the linear infill generation for the beam and bracket.

\subsection{FEA-influenced infill design (stage four)}

The fourth stage involves the generation of the infill design that has been influenced by the FEA results. To start, the linear infill design is taken from Stage Three and extended to include diagonal elements. This forms a lattice structure across the entire deposition layer and represents the potential elements that could be deposited by the FDM machine. To decide which elements are likely to provide the most benefit for the given loading, a weighting is applied to each element. This weighting is calculated by taking the average localised stress value from the FEA. Figure 12a provides an example of the potential elements with the weighting denoted by the shade of grey. 


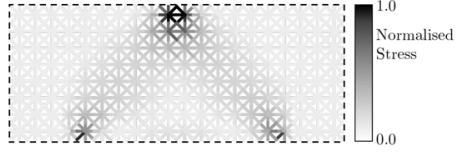

(a) Lattice mesh weighted by the local average stress from FEA

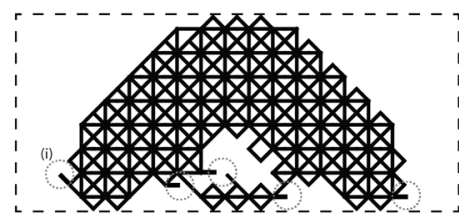

(b) Influenced infill design pre-pruning

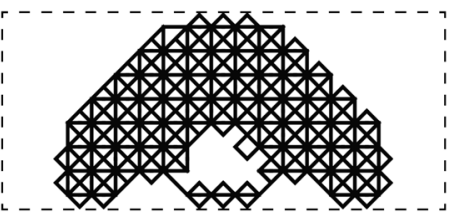

(c) Influenced infill design post-pruning

Fig. 12 Influenced infill design generation

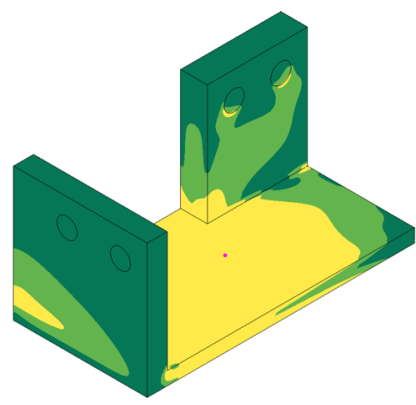

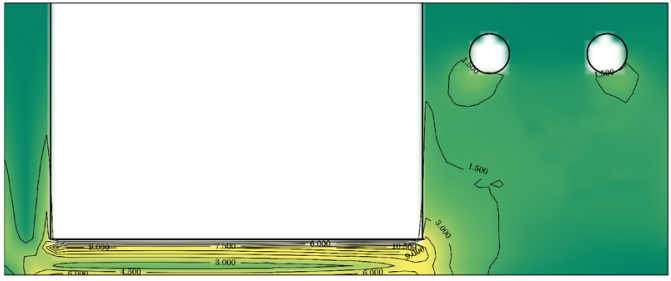

(a) FEA for bracket at $Z$ Level $5 \mathrm{~mm}$

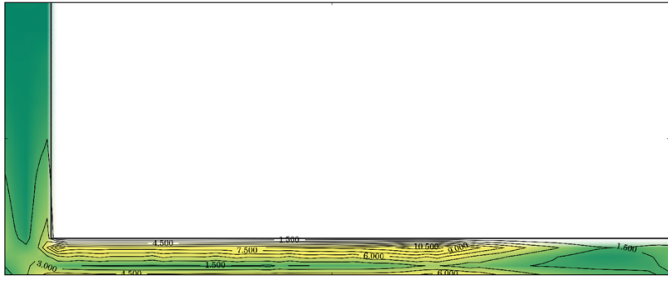

(c) FEA for bracket at Z Level $30 \mathrm{~mm}$

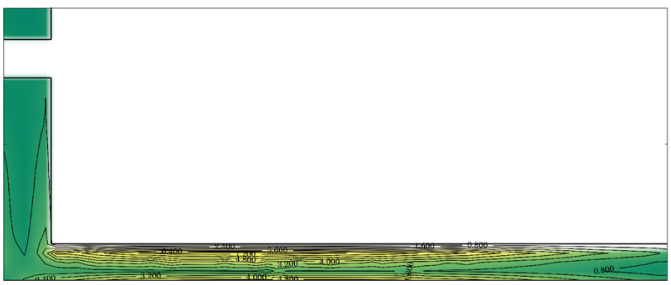

(e) FEA for bracket at Z Level $80 \mathrm{~mm}$

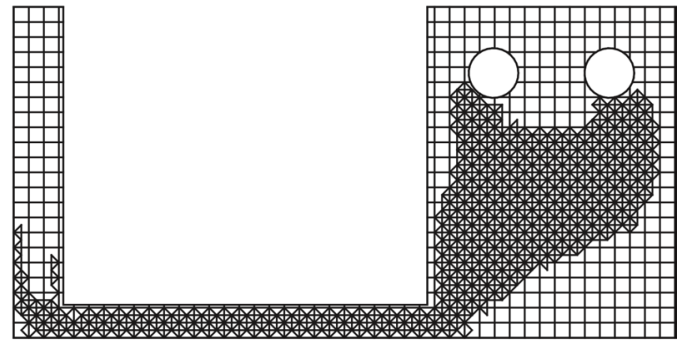

(b) optimised infill design

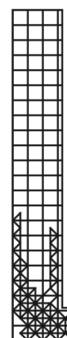

ฟH

(d) optimised infill design

囲

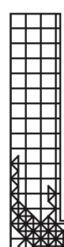

WHIIIIHI

(f) optimised infill design

Fig. 13 Strategies capability to handle a 3-dimensional stress case 


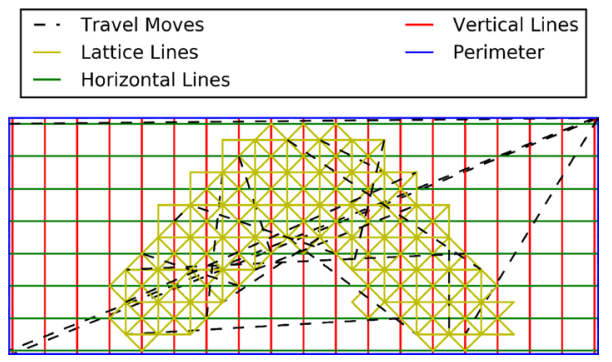

Fig. 14 Print line generation

The elements are then ordered by their stress values and the elements with least stress are removed until the desired infill percentage is attained (Fig. 12b). Following this, a post-process is performed on the infill design, where the remaining elements are analysed once more to remove elements that contain a single degree of connectivity (Fig. 12b, i). This is due to the fact that an element requires two degrees of connectivity for load transfer. Figure 12c shows the FEA-influenced infill design for the beam undergoing a three-point bend test with infill density of $50 \%$.

As this stage is performed for each layer, it enables the process to handle complex load cases. This is demonstrated in Fig. 13, which shows the infill for layers at 5, 30 and 80 $\mathrm{mm}$ for the bracket where it can be seen that the infill has been tailored to the relative stress distribution in each layer.

It is then the case of defining the deposition path for the layer where consideration has been made to minimise production time and ensure no overlapping of filament that would lead to distortions in the final printed component. First, the print paths for the linear infill are calculated. The process takes the vertical and horizontal paths and increments back and forth to minimise travel of the printer head. The FEA-influenced infill design is then mapped onto the linear infill structure. The elements within the FEA-influenced infill design are then compared to the linear infill design to remove any potential duplicate elements. The optimum print path for the remaining elements is then calculated. This is achieved by applying graph theory to detect whether the remaining elements form a Eulerian graph. If this is the case, the process solves the Chinese Postman Problem to generate a route that visits each element only once [32-34]. If the network is non-Eulerian, the process identifies the elements with odd connectivity. Travel moves are then added between these elements to ensure that they have an even degree of connectivity and thus, lead to an overall Eulerian graph. Figure 14 provides an example of the print lines generated for a layer of the three-point bend test beam.

The process then continues to loop through Stages Two, Three and Four to form the print lines for the entire part.
It is then a case of taking these print lines and forming the code that a FDM machine can use to manufacture the part.

\subsection{G-code generation (stage five)}

The fifth and final stage of the process involves the generation of the G-Code for the FDM printer. For each deposition layer, the perimeter path G-Code is generated, followed by the combined printer path from the merging of the linear and FEA-influenced infill designs. The pre- and post-amble G-Code for the specific FDM printer is then inserted, resulting in the final G-Code file.

\subsection{Summary}

This section has presented, in detail, a five-stage process that integrates the results of FEA into the infill generation of FDM parts. The key features of the strategy are that it takes the manufacturing constraints of FDM into account, does not alter the external geometry and is able to handle complex geometries and load cases.

Although the process has been automated where possible, there are a number of settings that are manually set by the user. These are:

Layer height $(\mathrm{mm})$ The increment in the z-axis.

Maximum mesh width ( $\mathrm{mm}$ ) The largest permissible mesh square for the part.

Minimum mesh width $(\mathrm{mm})$ The smallest permissible mesh square for the part.

Extrusion rate The length of material to be extruded per length of travel.

Mesh offset $(\mathrm{mm})$ The permissible gap between the perimeter and infill design.

Infill density The percentage of the interior volume that should be consumed by material.

\section{Investigating the potential of FEA-influenced infill designs}

To investigate the potential affordances of influencing the infill design using FEA, a beam undergoing four different loading scenarios has been tested. The test cases are as follows with further details presented in Fig. 15.

1. Three-point bend test.

2. Off-centre three-point bend test.

3. Four-point bend test.

4. Inverted four-point bend test.

The experimental procedure is consistent with the work by Lužanin et al. [26] who performed a three-point bend test to 
Fig. 15 Illustration of test piece and image of test case

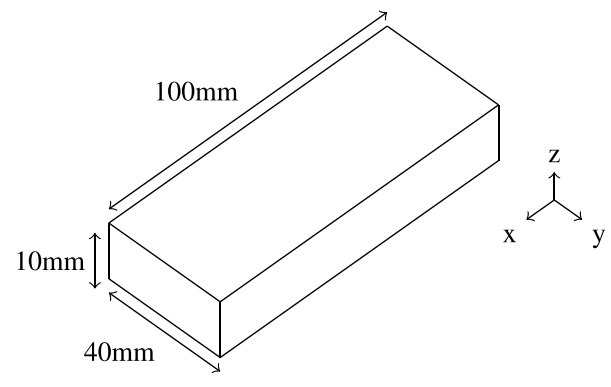

(a) Beam geometry

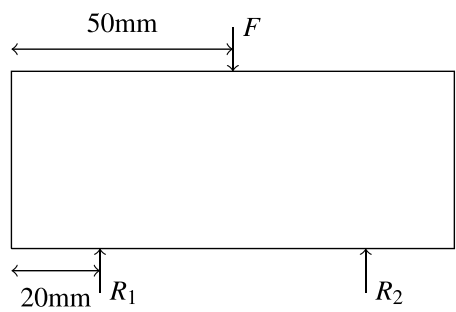

(c) Three-point bend test

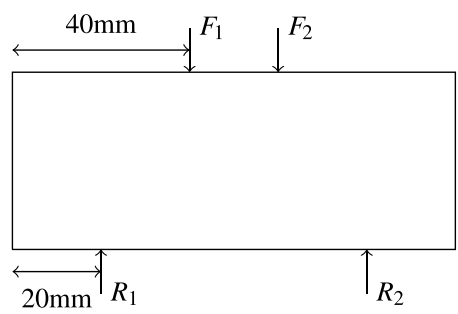

(e) Four-point bend test

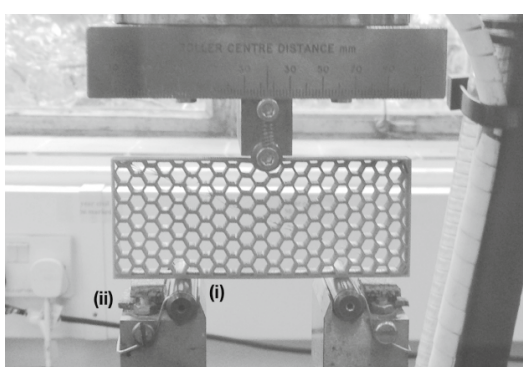

(b) Test machine

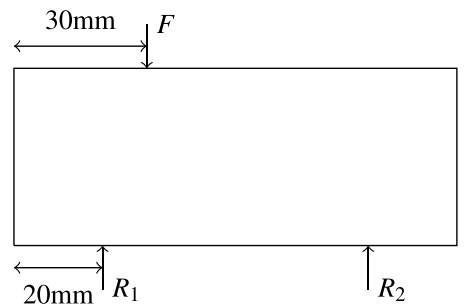

(d) Offset three-point bend test

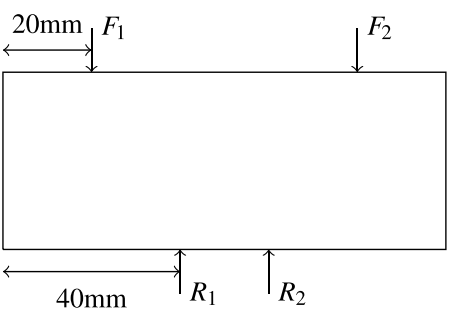

(f) Inverted four-point bend test evaluate the effect of layer thickness, deposition angle and infill percentage on the maximum flexural strength of FDM parts. For this investigation, beams of $100 \mathrm{~mm}$ length, 40 $\mathrm{mm}$ height and $10 \mathrm{~mm}$ in depth have been tested on a $25 \mathrm{kN}$ Instron machine (see, Fig. 15). The Instron machine was set to provide a constant linear displacement of $0.02 \mathrm{mms}^{-1}$ in the direction of compression and the compression continued until the specimen either destructed or interfered with the

Table 1 Material extrusion details for test pieces

\begin{tabular}{ll}
\hline Beam & Value $(\mathrm{mm})$ \\
\hline Reference & \\
$\quad$ Honeycomb & 4289.4 \\
Optimised & \\
Three-point bend test & $4251.2(-0.9 \%)$ \\
Off-centre three-point bend test & $4224.6(-1.5 \%)$ \\
Four-point bend test & $4285.6(+0.09 \%)$ \\
Inverted four-point bend test & $4234.3(-1.3 \%)$ \\
Statistics & \\
Mean & 4257.0 \\
Standard deviation & 26.3 \\
\hline
\end{tabular}

testing equipment. For example, where the beam was no longer being supported by the rollers (Fig. 15b, i) but the shoulders of the jig itself (Fig. 15b, ii). The specimens were tested to their stress-strain limits so that comparisons across the full extent of their structural behaviour can be made. The test was repeated five times for each case.

For each test case, the industry standard hexagonal pattern design formed the baseline against which the FEAinfluenced infill design was compared. To further control the conditions of the tests, the level of extruded material remained constant and reflects a $32 \%$ infill density for all beams concerned. Table 1 provides further details on the amount of deposited material with the maximum difference between the reference specimen and a test specimen being no more than $1.5 \%$ in favour of the reference specimen. The material used was a polylactic acid (PLA) as sold by MakerBot Industries $^{2}$ with a $1.75 \mathrm{~mm}$ diameter filament.

The slicing and generation of the infill has been based on the $x$-, $y$-plane as highlighted in Fig. 15a. Figure 16 illustrates the FEA-influenced infill designs that have

\footnotetext{
${ }^{2}$ http://downloads.makerbot.com/filament/PLA+MSDS.pdf.
} 


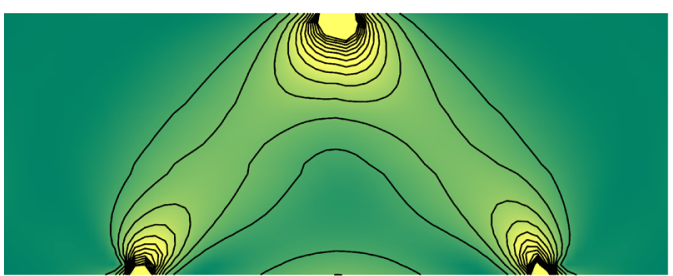

(a) Three-Point Bend Test FEA

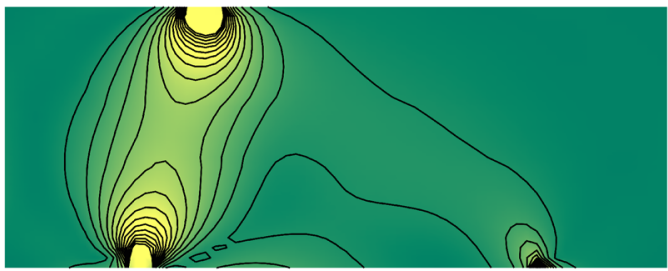

(c) Off-Centre Three-Point Bend Test FEA

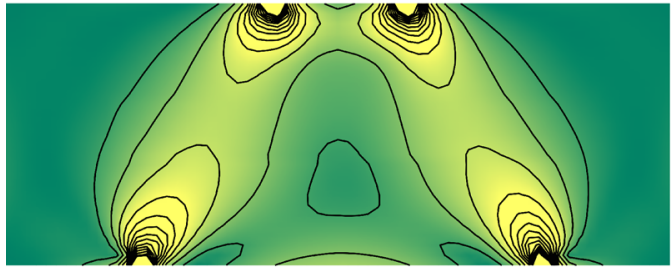

(e) Four-Point Bend Test FEA

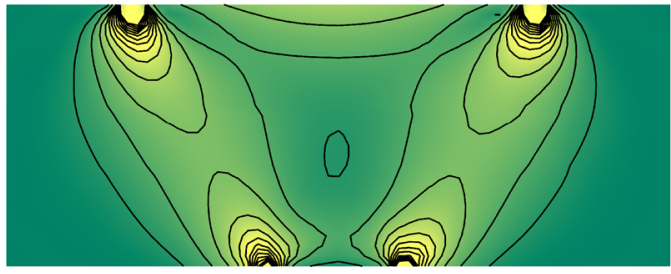

(g) Inverted Four-Point Bend Test FEA

Fig. 16 Finite element results and associated test pieces

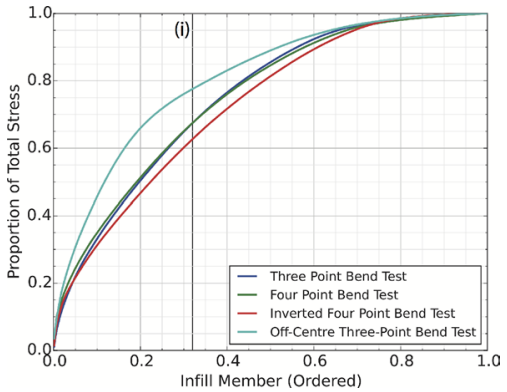

Fig. 17 Beam stress along potential lattice elements

been generated whilst Fig. 17 shows the Pareto chart for the lattice elements in terms of their weightings that have been calculated during Stage Four of the process. The lattice elements have been ordered by their contribution. A steeper curve indicates that fewer elements are required

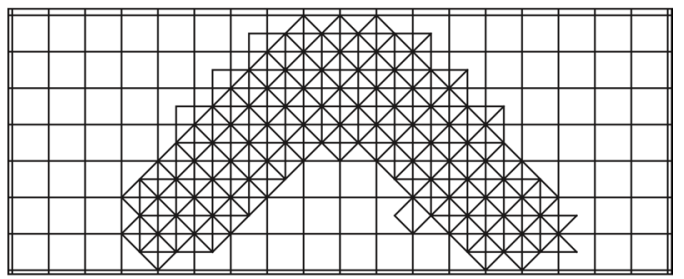

(b) Three-Point Bend Test Infill Structure

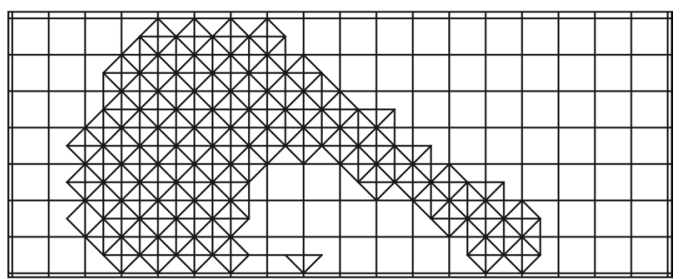

(d) Off-Centre Three-Point Bend Test Infill Structure

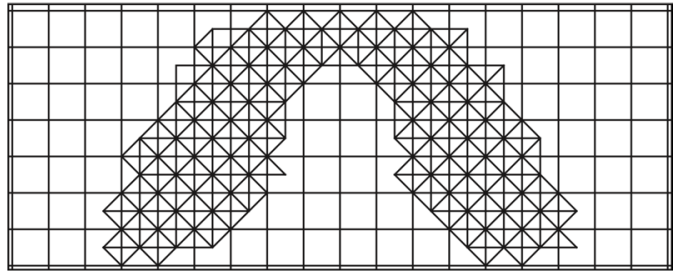

(f) Four-Point Bend Test Infill Structure

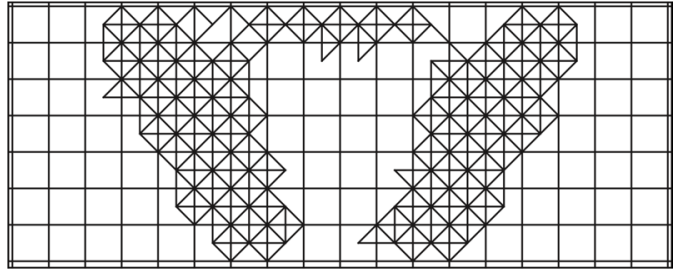

(h) Inverted Four-Point Bend Test Infill Structure

to carry the majority of the load and thus, there is greater potential in increasing the strength-to-weight ratio of the component if the non-load-carrying lattice elements are removed.

The vertical line (1) highlights the cut-off to ensure a $32 \%$ infill density for all designs. It can be seen that the offcentre three-point bend test has the potential to benefit most in terms of strength-to-weight ratio as approximately $78 \%$ of the cumulative weighting is carried by the lattice elements that have remained in the FEA-influenced infill design. Similarly, it can be asserted that the inverted four-point bend test will see a reduced benefit as the weightings are more evenly distributed across the test piece and the remaining lattice elements cover just $65 \%$ of the imposed stress. The remaining three-point and four-point bend specimens are likely to experience a similar change to the strength of the material as $68 \%$ of total internal stresses are covered by the remaining lattice elements. 
Table 2 Max force, extension and stiffness table of results

\begin{tabular}{|c|c|c|c|c|}
\hline & \multicolumn{2}{|l|}{ Three-point } & \multicolumn{2}{|c|}{ Off-centre three-point } \\
\hline & Honeycomb & Optimised & Honeycomb & Optimised \\
\hline \multicolumn{5}{|l|}{$\operatorname{Max}$ force $(\mathrm{N})$} \\
\hline Mean & 534.6 & $995.6(1.9 \times)$ & 459.1 & $1633.1(3.5 \times)$ \\
\hline Standard deviation & 85.1 & $92.1(1.0 \times)$ & 63.7 & $225.0(3.5 \times)$ \\
\hline \multicolumn{5}{|c|}{ Max force extension (mm) } \\
\hline Mean & 14.4 & $6.3(0.4 \times)$ & 13.6 & $13.7(1.0 \times)$ \\
\hline Standard deviation & 5.1 & $0.6(0.1 \times)$ & 2.5 & $2.3(0.9 \times)$ \\
\hline \multicolumn{5}{|l|}{ Stiffness $\left(\mathrm{N} / \mathrm{mm}^{2}\right)$} \\
\hline Mean & 151.9 & $492.3(3.2 \times)$ & 144.3 & $298.0(2.1 \times)$ \\
\hline Standard deviation & 9.7 & $32.1(3.3 \times)$ & 36.4 & $53.1(1.5 \times)$ \\
\hline \multicolumn{5}{|c|}{ Energy absorbed ( $\mathrm{N} \mathrm{mm})$} \\
\hline Mean & $6,013,261.2$ & $8,448,650.0(1.4 \times)$ & $5,005,817.1$ & $15,287,407.5(3.1 \times)$ \\
\hline \multirow[t]{3}{*}{ Standard deviation } & $398,381.9$ & $2,658,821.2(6.6 \times)$ & $248,686.6$ & $1,469,032.7(5.9 \times)$ \\
\hline & \multicolumn{2}{|l|}{ Four-point } & \multicolumn{2}{|c|}{ Inverted four-point } \\
\hline & Honeycomb & Optimised & Honeycomb & Optimised \\
\hline \multicolumn{5}{|l|}{ Max force $(\mathrm{N})$} \\
\hline Mean & 721.1 & $972.7(1.3 \times)$ & 673.2 & $1206.2(1.8 \times)$ \\
\hline Standard deviation & 154.2 & $131.8(1.1 \times)$ & 77.5 & $135.3(1.7 \times)$ \\
\hline \multicolumn{5}{|c|}{ Max force extension (mm) } \\
\hline Mean & 14.4 & $6.3(0.4 \times)$ & 13.6 & $13.7(1.0 \times)$ \\
\hline Standard deviation & 8.8 & $1.9(0.2 \times)$ & 2.6 & $2.4(0.92 \times)$ \\
\hline \multicolumn{5}{|l|}{ Stiffness $\left(\mathrm{N} / \mathrm{mm}^{2}\right)$} \\
\hline Mean & 187.4 & $345.1(1.8 \times)$ & 213.0 & $252.5(1.2 \times)$ \\
\hline Standard deviation & 60.4 & $47.5(0.8 \times)$ & 56.5 & $76.6(0.74 \times)$ \\
\hline \multicolumn{5}{|l|}{ Energy absorbed ( $\mathrm{N} \mathrm{mm})$} \\
\hline Mean & $7,054,588.9$ & $5,055,034.9(0.7 \times)$ & $7,555,013.6$ & $13,577,009.8(1.8 \times)$ \\
\hline Standard deviation & $335,315.7$ & $3,985,400.9(11.9 \times)$ & $1,039,383.9$ & $1,348,942.1(1.3 \times)$ \\
\hline
\end{tabular}

\section{Results and discussion}

To evaluate the proposed process, the stress-strain curves and the co-variance of the maximum load and displacement at which it occurred have been plotted. In addition, the mean, variance and standard deviation for the maximum force and stiffness have been calculated. The stiffness has been calculated for the gradient between the $0-0.8 \mathrm{~mm}$ displacement for all specimens.

The results from the four test scenarios have been combined in Fig. 18 and Table 2. The graphs show the stress-strain and co-variance plots of the maximum loading for the FEA-influenced and hexagonal infill designs. The superimposed ellipses represent standard deviations from the mean point of failure. An ideal case would be a small elliptical shape, which would indicate that the specimens have a consistent and predictable failure mode. The colour of the traces corresponds to the specimen drawings within the legend.
The main objective of the evaluation is to understand the effect the infill design process has had on the structural behaviour of the component across its entire stress-strain range and thus, the following sections present and discuss the results from the test cases in relation to the:

1. Maximum loading capacity;

2. Extension at which maximum loading occurs;

3. Beam stiffness;

4. Energy absorption; and;

5. Failure modes of the structures.

\subsection{Maximum loading capacity}

Focusing on the maximum loading capacity of the beams tested, it is immediately apparent from Fig. 18 that the FEAinfluenced infill design can accommodate a much greater loading than the hexagonal. In particular, the off-centre three-point bend test sees the greatest increase in loading capacity with a three-fold increase whilst the four-point bend 
Fig. 18 Stress-strain and maximum load distribution of test specimens
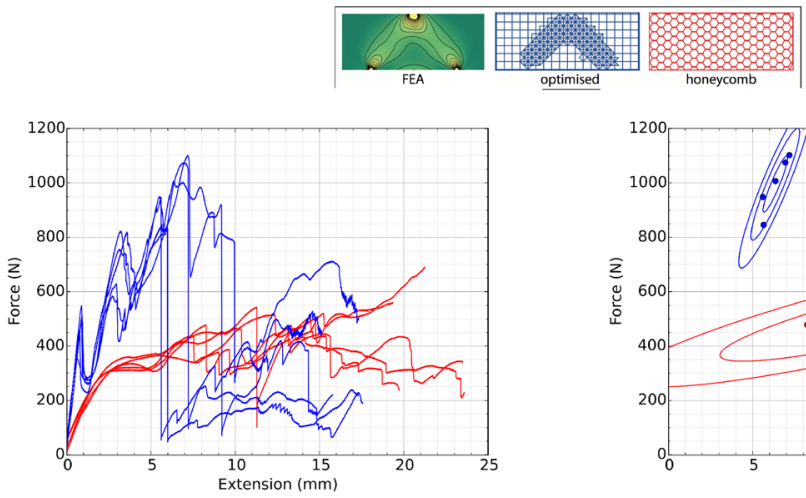

(a) Three-point bend test stress-strain
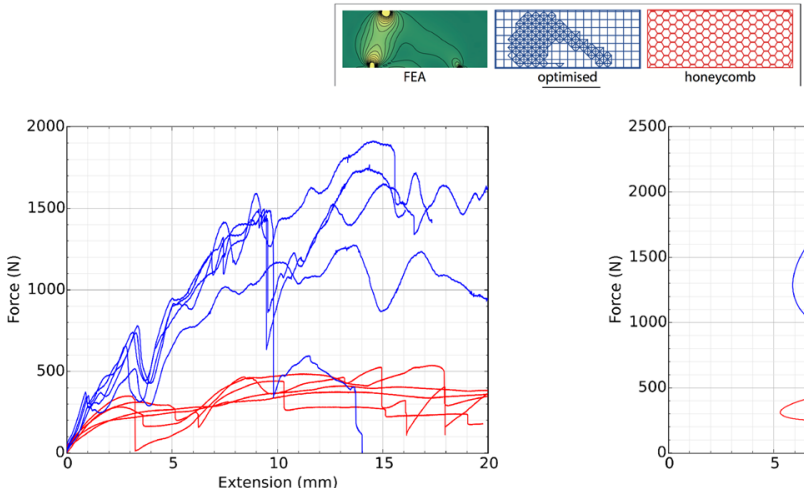

(c) Off-centre three-point bend test stress-strain

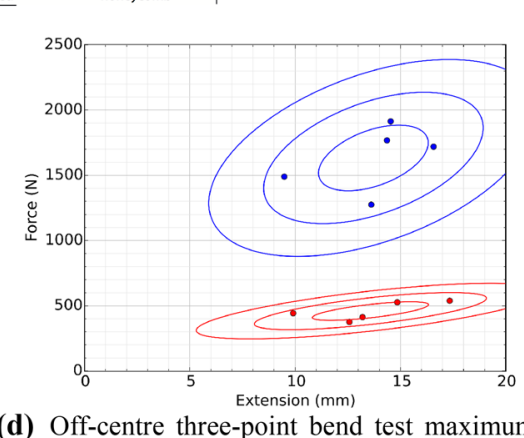

(d) Off-centre three-point bend test maximum loading
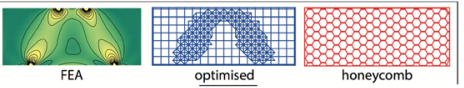

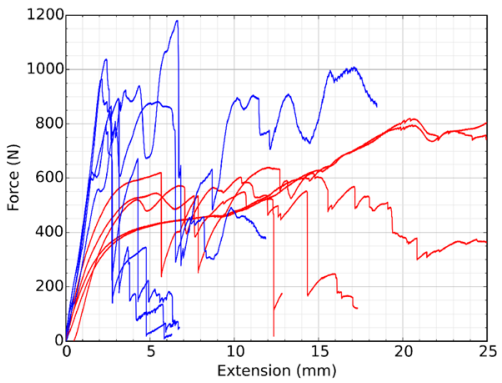

(e) Four-point bend test stress-strain

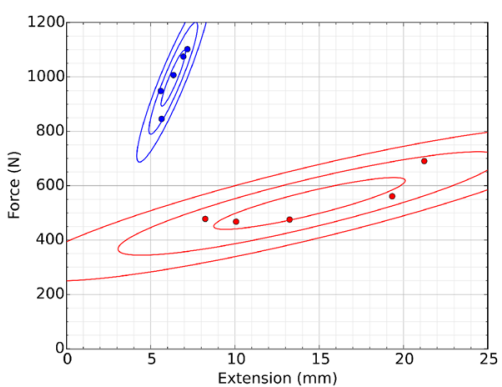

(b) Three-point bend test maximum loading

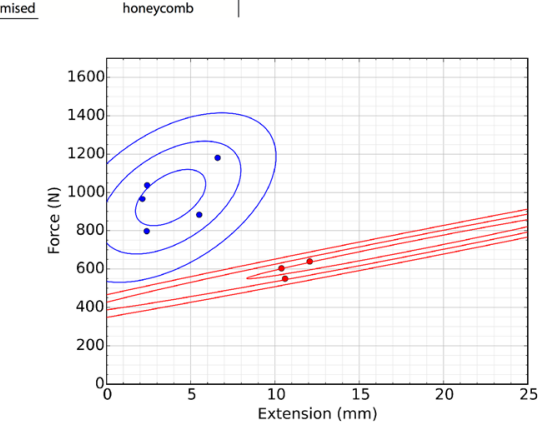

(f) Four-point bend test maximum loading
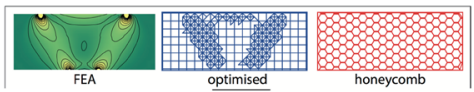

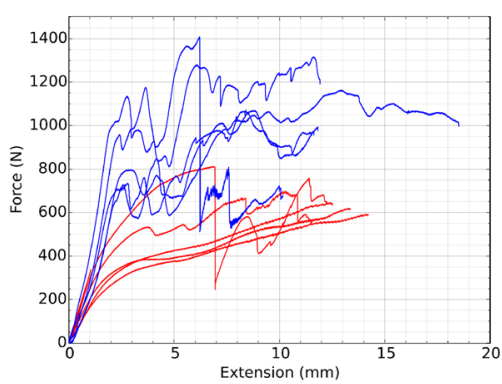

(g) Inverted four-point bend test stress-strain

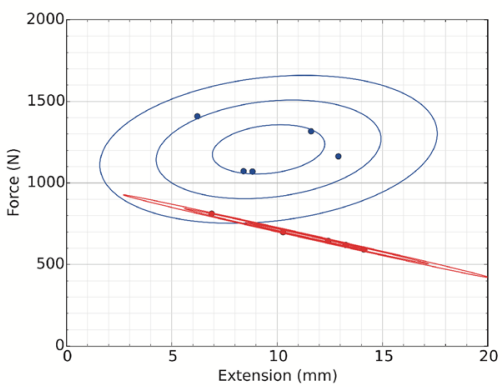

(h) Inverted four-point bend test maximum loading 
test sees the least gain of 30\%. This relative level of benefit is consistent with the relationship between the proportion of stress contained by the remaining paths in the FEA-influenced infill designs (Fig. 17).

Although the off-centre three-point beam shows the greatest increase in maximum loading capacity, it also shows the greatest variance in loading capacity. This suggests that the more defined the stress profile, the more susceptible the infill design is to variations in the testing of the beam. It is also the case that the variance of the optimised beams is consistently greater than the variance of the hexagonal beams across the four test cases.

\subsection{Maximum force extension}

Considering the extension at which the maximum force occurs, it can be seen from Table 2 that the maximum force occurs at either an equivalent or lower strain for the FEAinfluenced infill when compared to the hexagonal. Although, it is interesting to note that the beam that experienced the least benefit in maximum force (four-point bend test) sees the greatest reduction in the strain at which the force occurs. In contrast, the off-centre three-point test that exhibited the greatest increase in maximum force saw comparably very little change in strain at which the maximum occurred. Thus, it appears there is a degree of negative correlation between the maximum stress and strain values for the FEA-influenced infill designs.

In contrast to the maximum loading condition, the variance of the strain level at which the maximum loading occurs is more consistent for the optimised beams than for the hexagonal beams. This could be an important feature if one were to use these designs for particular failure modes and require a component to fail at a particular strain. This observation holds true across all four test cases, where the variance in strain appears consistent and provides further evidence of the potential utility for using the infill design for designing components that require specific operating strains.

\subsection{Beam stiffness}

The average and standard deviations for the stiffness for the beams is also presented in Table 2. In all four test cases, the FEA-influenced infill designs consistently outperformed the hexagonal infill designs exhibiting up to three times the stiffness with the maximum gain being achieved during the three-point bend test. This also highlights the significance of the loading scenario when investigating topology optimisations as the greatest changes in maximal loading, strain at which maximal occurs and stiffness have been measured during different loading scenarios.

In contrast to the maximum force and strain metrics, the stiffness exhibited by the beams is relatively consistent when repeated. This provides an indication that the initial response behaviour of FDM beams is consistent within the elastic deformation region and it is the plastic region where deviations in the stress-strain behaviour of parts with the same infill design occurs.

\subsection{Energy absorption}

The final aspect of the results presented in Table 2 are the energy absorption characteristics. All but one case demonstrated an increase in energy absorption for the optimised beam with the off-centre three-point beam showing the greatest increase and four-point showing a decrease in energy absorption. This can be attributed to the premature failure of the four-point beam whilst the honeycomb design was able to sustain a much greater extension. This further confirms that components with a well-defined load profile benefits most from an FEA-influenced infill design.

Focusing on the standard deviation across the test cases, the FEA-influenced infill designs reveal a greater deviation in their energy absorption capacity. Thus, even though there is a general increase in the energy absorption capacity of the component for the given loading scenario, it is more difficult to determine and predict the absorption capacity for the optimised beam.

\subsection{Failure modes of the structures}

When considering the failure modes, there are some interesting buckling phenomena that are exhibited across both the FEA-influenced and hexagonal infill designs. In increasing the stiffness of the beam through the FEA-influenced design, the brittleness of the beam also increases. With each element of the FEA-influenced structure contributing significantly to the load-carrying capacity of the beam, it can be seen from Fig. 18a that any buckling or element failure leads to a substantial decrease in the load-carrying performance. This is particularly apparent in the three-point and off-centre threepoint bend tests (Fig. 18a, c).

There are also consistent buckling events that occur in the early stages of the loading of the FEA-influenced beams and this is where the pathways connecting the infill design and perimeter buckle. The rollers of the jig then settle within the optimised structure where the load-carrying capacity starts to increase once again (Fig. 19b). The FEA-influenced infill then completely fails after reaching the maximum loading condition (Fig, 19c). The consistency of the buckling points for the FEA-influenced beams could be used to support planned and predictable failure modes for parts, which could also help in post-failure analysis.

In contrast, the hexagonal beams begin to plateau at a steady-state loading condition. The beams would then steadily deform whilst maintaining this load before failing or 
Fig. 19 Buckling and failure modes of the beams designs

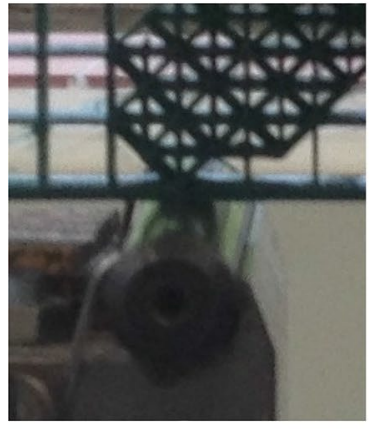

(a) Pre-Buckle

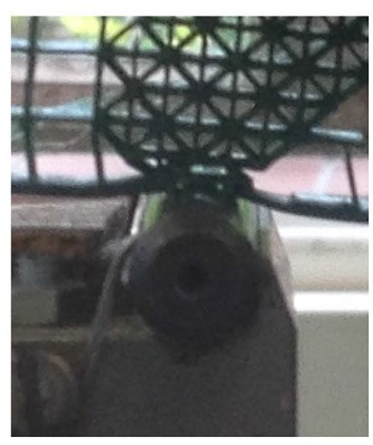

(b) Post-Buckle

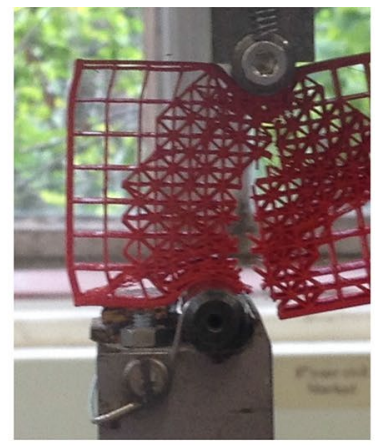

(c) Sudden failure of optimised infill design

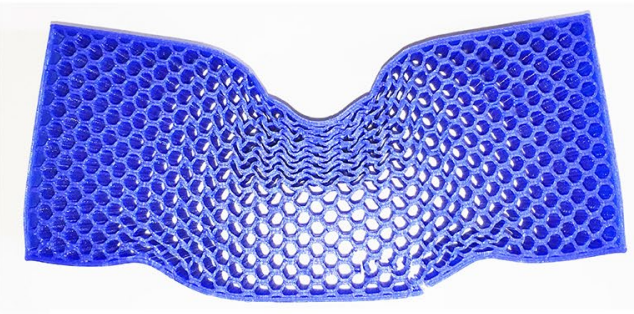

(d) Layers formed by buckled hexagonal infill

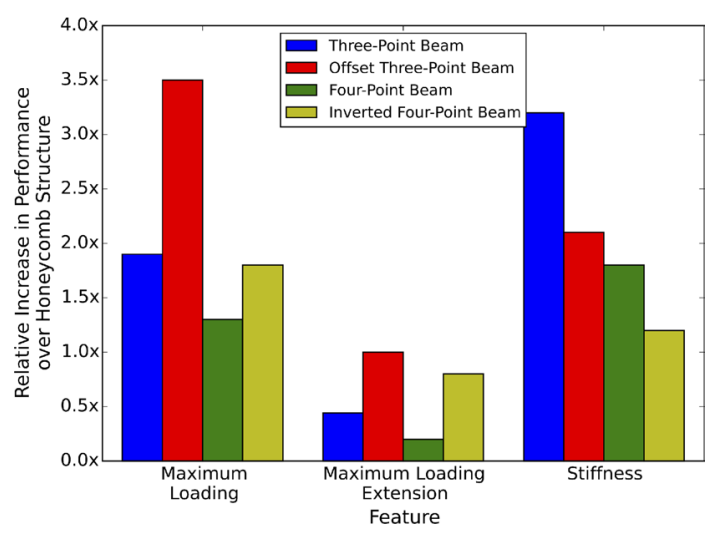

Fig. 20 Comparison of key loading features from the four test cases

reaching a stage where the hexagonal structure began forming layers of deformed material (Fig. 19d). In this case, the loading would often increase as the beam starts to form an almost solid structure.

\subsection{Summary}

From the four test cases, five key findings are evidenced with Fig. 20 highlighting the key results from each of the tests. The first is that in all cases, the FEA-influenced infill design outperforms the hexagonal structure in supporting higher loads. Second, the FEA-influenced infill provided substantial increases in strength in situations where the stress profile is less distributed across the part to be printed. In addition, the extension at which the maximum loading occurred was reduced for the FEA-influenced infill. This leads onto the third finding where the FEA-influenced infill are stiffer than the hexagonal design. The fourth finding is that the FEA influenced infill designs generally saw a reduction in the co-variance at which the maximum loading point occurred. This is an indicator that the FEA-influenced infill is more predictable in its loading behaviour.

The fifth finding is the predictability of the loading behaviour has also been seen in how the parts begin to buckle. The FEA-influenced infill design saw a consistent perimeter buckling event, which commonly occurred at the same level of displacement. Embodying this predictability of buckling could enable designers to use the buckling behaviour as preemptive warning signs for particular load limits.

\section{Future work}

This paper has demonstrated the potential of using FEA to influence the infill design process of FDM-printed parts where an increase loading capacity of four beams in different loading conditions has been observed. It has also explored the generalisability of the proposed process through its ability to handle complex geometries such as the bracket (c.f. Fig. 13). In the creation of the proposed process, a number of new research challenges have been exposed.

First, the process has only been evaluated using twodimensional loading cases and there is a need to understand the potential of the strategy for more complex 
loading scenarios. This includes three-dimensional load cases, which involve varying levels of compression, tension, torsion and/or a mixture of modes. In addition, cyclic loading and/or fatigue testing could be considered. Being able to standardise these tests would also enable comparison between a wider range of alternative infill designs.

Second, for the tests presented, the volume of material was controlled but it has been shown that the best performing design occurred where the number of structural paths covered the majority of the internal stress distribution within the beam (i.e. the off-centre three-point beam test). It would be interesting to see how an improved strength-to-weight ratio can be determined by the stress profile of the beam alone and to evaluate the optimisation across a range of infill densities.

Third, although the FEA-influenced design has been shown to improve the loading capacity of the beam, there is currently no computational method to predict the improvement. In addition, the current FEA model is used solely to guide the design of the infill and does not reflect the actual stresses the internal geometry is likely to experience. Thus, future research could post-process the print file to predict the loading and buckling profile of the part ahead of printing. This could also lead to an iterative procedure where incremental changes to the lattice structure could be made and evaluated computationally to arrive at further improved internal structure.

Fourth, the optimisation has only considered a lattice design and there exists many other configurations that could be used to generate the optimised infill design. Such designs could potentially use the honeycomb as the base pattern and/or adapt meshing techniques used in FEA and CFD and structures developed in the natural world. In addition, the strategy does not take the material into account and one of the key areas of development in FDM is the ability to print multiple materials. Future processes could, therefore, seek to determine the optimum placement of combinations of materials to achieve the desired mechanical properties for a component.

Related to the aforementioned area is the challenge of determining the attributes of the infill design that contribute to the overall performance gains in the component. Future work could investigate whether the performance gains were due to:

- higher material concentrations in the stressed regions of the component;

- the orientation of the lattice elements; and/or,

- the anisotropic behvaiour of the material.

\section{Conclusion}

Additive manufacturing (AM) has and continues to experience considerable market and technological growth with many forecasting a tripling in market value in the next decade. A key affordance of these technologies has been the increased design freedom in both the external form and internal structure. This paper has taken advantage of this affordance and uses the results from FEA to influence the infill design of FDM parts.

This process has been discussed in depth alongside two cases that of a beam and bracket undergoing contrived loading scenarios. A key feature of the proposed process is its ability to generate FEA-influenced infills for complex parts with multiple perimeters and three-dimensional loading conditions.

The proposed process has been evaluated experimentally for a beam in four different loading cases and the designs generated were compared to the industry standard honeycomb infill. These tests confirmed a significant performance increase of the beam under loading, where the tests showed a:

- $3.5 \times$ increase in the loading capacity of the beam, particularly for highly localised stress profiles;

- $3 \times$ increase in the stiffness of the beam;

- reduction in the co-variance of the maximum loading case; and,

- more predictable buckling mode.

In creating the proposed process, four research areas were exposed and are summarised as:

1. further evaluations involving three-dimensional, compressive, tensile, torsional and cyclic load cases leading to a standardised testing platform for print parts;

2. further optimisation of the beam structuring through self-determination of the optimum infill percentage;

3. post-processing of part geometry to print to predict loadcarrying capacity and loading/buckling profile; and,

4. further analysis of potential infill designs including the use of multiple materials within the part.

Acknowledgements The work reported in this paper has been undertaken as part of the Language of Collaborative Manufacturing Project at the University of Bath and University of Bristol, which is funded by the Engineering and Physical Sciences Research Council (EPSRC), Grant reference EP/K014196/2. Underlying data are openly available from the University of Bath Research Data Archive at https://doi. org/10.15125/BATH-00420 and a demonstration of the process can be found at https://nbviewer.jupyter.org/github/JamesGopsill/PythonNotebooks/blob/master/FDM\%20Force\%20Path\%20Methods/Generating $\% 20$ infill $\% 20$ designs $\% 20$ based $\% 20$ on $\% 20$ forces $\% 20$ through $\% 20$ a\%20part.ipynb. 


\section{Compliance with ethical standards}

Conflict of interest On behalf of all authors, the corresponding author states that there is no conflict of interest.

Open Access This article is distributed under the terms of the Creative Commons Attribution 4.0 International License (http://creativecommons.org/licenses/by/4.0/), which permits unrestricted use, distribution, and reproduction in any medium, provided you give appropriate credit to the original author(s) and the source, provide a link to the Creative Commons license, and indicate if changes were made.

\section{References}

1. Gonigam D (2013) Printed to life: the tipping point. http://agorafinancial.com/2013/11/12/printed-to-life-the-tipping-point/. Accessed 01 Aug 2016

2. Palermo E (2013) Fused deposition modeling: most common 3d printing method. http://www.livescience.com/39810-fused-deposition-modeling.html. Accessed 01 Aug 2016

3. Rozvany G (2009) A critical review of established methods of structural topology optimization. Struct Multidiscip Optim 37(3):217. https://doi.org/10.1007/s00158-007-0217-0

4. Sigmund O, Maute K (2013) Topology optimization approaches. Struct Multidiscip Optim 48(6):1031. https://doi.org/10.1007/ s00158-013-0978-6

5. Deaton JD, Grandhi RV (2014) A survey of structural and multidisciplinary continuum topology optimization: post 2000. Struct Multidiscip Optim 49(1):1. https://doi.org/10.1007/ s00158-013-0956-Z

6. Gopsill JA, Hicks BJ (2016) Deriving infill design of fused deposition modelled parts from predicted stress profiles In: Proceedings of the ASME 2016 International Design Engineering Technical Conferences \& Computers and Information in Engineering Conference (Charlotte, North Carolina, USA, 2016)

7. Lu L, Sharf A, Zhao H, Wei Y, Fan Q, Chen X, Savoye Y, Tu C, Cohen-Or D, Chen B (2014) Build-to-last: strength to weight 3D printed objects. ACM Trans Gr 33(4):97:1. https://doi. org/10.1145/2601097.2601168

8. Tomlin M, Meyer J (2011) Topology optimization of an additive layer manufactured (ALM) aerospace part. In: Proceeding of the 7th Altair CAE technology conference, pp 1-9

9. Meisel N, Williams C (2015) An investigation of key design for additive manufacturing constraints in multimaterial three-dimensional printing. J Mech Des 137(11):111406

10. Ranscombe C, Hicks B, Mullineux G, Singh B (2012) Visually decomposing vehicle images: exploring the influence of different aesthetic features on consumer perception of brand. Des Stud 33(4):319

11. Ahn SH, Montero M, Odell D, Roundy S, Wright PK (2002) Anisotropic material properties of fused deposition modeling ABS. Rapid Prototyp J 8(4):248. https://doi. org/10.1108/13552540210441166

12. Sood AK, Ohdar R, Mahapatra S (2009) Improving dimensional accuracy of fused deposition modelling processed part using grey taguchi method. Mater Des 30(10):4243. https://doi.org/10.1016/j. matdes.2009.04.030

13. Lanzotti A, Del Giudice DM, Lepore A, Staiano G, Martorelli M (2015) On the geometric accuracy of RepRap open-source threedimensional printer. J Mech Des 137(10):101703

14. Pennington RC, Hoekstra NL, Newcomer JL (2005) Significant factors in the dimensional accuracy of fused deposition modelling. Proc Inst Mech Eng Part E J Process Mech Eng 219(1):89. https:// doi.org/10.1243/095440805X6964
15. Gregorian A, Elliot B, Navarro R, Ochoa F, Singh H, Monge E, Foyos J, Noorani R, Fritz B, Jayanthi S (2001) Accuracy improvement in rapid prototyping machine (FDM-1650). In: Solid Freeform Fabrication Proceedings, pp 77-84

16. Zhang Y, Chou K (2008) A parametric study of part distortions in fused deposition modelling using three-dimensional finite element analysis. Proc Inst Mech Eng Part B J Eng Manuf 222(8):959

17. Pandey P, Reddy N, Dhande S (2003) Real time adaptive slicing for fused deposition modelling. Int J Mach Tools Manuf 43(1):61. https://doi.org/10.1016/S0890-6955(02)00164-5

18. Leary M, Merli L, Torti F, Mazur M, Brandt M (2014) Optimal topology for additive manufacture: a method for enabling additive manufacture of support-free optimal structures. Mater Des 63:678. https://doi.org/10.1016/j.matdes.2014.06.015

19. Rezaie R, Badrossamay M, Ghaie A, Moosavi H (2013) Topology optimization for fused deposition modeling process. Procedia CIRP 6:521. https://doi.org/10.1016/j.procir.2013.03.098

20. Ziemian C, Sharma M, Ziemian S (2012) Anisotropic mechanical properties of ABS parts fabricated by fused deposition modelling, Mechanical Engineering. In: Gokcek M (ed) InTech. https://doi. org/10.5772/34233

21. Es-Said OS, Foyos J, Noorani R, Mendelson M, Marloth R, Pregger BA (2000) Effect of layer orientation on mechanical properties of rapid prototyped samples. Mater Manuf Process 15(1):107. https://doi.org/10.1080/10426910008912976

22. Ghorpade A, Karunakaran K, Tiwari M (2007) Selection of optimal part orientation in fused deposition modelling using swarm intelligence. Proc Inst Mech Eng Part B J Eng Manuf 221(7):1209

23. Sood AK, Ohdar R, Mahapatra S (2010) Parametric appraisal of mechanical property of fused deposition modelling processed parts. Mater Des 31(1):287. https://doi.org/10.1016/j.matdes.2009.06.016

24. Jones RO, Iravani P, Bowyer A (2012) Rapid manufacturing of functional engineering components. Other, Bath, UK. http://opus. bath.ac.uk/30281/

25. Rayegani F, Onwubolu GC (2014) Fused deposition modelling (FDM) process parameter prediction and optimization using group method for data handling (GMDH) and differential evolution (DE). Int J Adv Manuf Technol 73(1-4):509

26. Lužanin O, Movrin D, Plančak M (2014) Effect of layer thickness, deposition angle, and infill on maximum flexural force in FDMbuilt specimens. J Technol Plast 39(1):49-58

27. Onwubolu GC, Rayegani F (2014) Characterization and optimization of mechanical properties of ABS parts manufactured by the fused deposition modelling process. Int J Manuf Eng 2014. https:// doi.org/10.1155/2014/598531

28. Lei N, Yao X, Moon SK, Bi G (2016) An additive manufacturing process model for product family design. J Eng Des 27(11):751. https://doi.org/10.1080/09544828.2016.1228101

29. Yang X, Xie Y, Steven G (2005) Evolutionary methods for topology optimisation of continuous structures with design dependent loads. Comput Struct 83(12):956

30. Eschenauer HA, Olhoff N (2001) Topology optimization of continuum structures: a review. Appl Mech Rev 54(4):331

31. Schroeder WJ, Lorensen B, Martin K (2004) The visualization toolkit, 4th edn. Kitware

32. Algorithms and Theory of Computation Handbook, CRC Press LLC, 1999, "Chinese postman problem", in Dictionary of Algorithms and Data Structures [online], Pieterse V and Black PE, (eds.) 2 Sept 2014. Available from: https://www.nist.gov/dads/ HTML/chinesePostman.html. Accessed 10 June 2016

33. Kwan MK (1962) Graphic programming using odd or even points. Chin Math 1(273-277): 110

34. Hagberg AA, Schult DA, Swart PJ (2008) Exploring network structure, dynamics, and function using NetworkX. In: Proceedings of the 7th Python in Science Conference (SciPy2008), Pasadena, CA USA, pp 11-15 